\title{
Construction of Analytic, Unitary Scattering Amplitudes from a Given Differential Cross-Section: A Refined Analysis*
}

\author{
D. Atkinson
}

Institut voor Theoretische Natuurkunde, Groningen, Netherlands

\author{
P. W. Johnson and R. L. Warnock \\ Illinois Institute of Technology, Chicago, Illinois, USA
}

Received March 12, 1973

\begin{abstract}
We extend previous work concerning the construction of unitary scattering amplitudes that correspond to the scattering data at a given energy. The dispersive and absorptive parts are by construction analytic in $\cos \theta$ in the small and large Lehmann ellipses, respectively. The dispersive and absorptive parts obtained here, in contrast to those obtained before, are shown to have continuous derivatives on the boundary of their domains of analyticity. The continuum ambiguity in the determination of the scattering amplitude, which is associated with a lack of experimental information on the inelastic contribution to unitarity, is present here as well.
\end{abstract}

\section{Section I: Introduction}

The problem of determining the scattering amplitude $f$ at a given energy, from the differential cross-section $\sigma$ at that energy, has been considered by several authors recently [1]. In particular, Atkinson, Mahoux, and Ynduráin [2] have dealt with the problem of constructing a scattering amplitude $f(z)$ which is analytic in $z$, the cosine of the barycentric scattering angle, inside a certain unifocal ellipse. This amplitude must correspond to a specified differential cross-section $\sigma(z)$, which is analytic inside the ellipse; in addition, $f(z)$ must satisfy unitarity. The present work is a refinement of Ref. [2]; we discuss the same nonlinear equation as was treated there, but we do the analysis in a smaller Banach space and obtain stronger results.

For simplicity, we limit our discussion in the first two sections to the case of purely elastic unitarity. The results may readily be generalized to handle a fixed contribution to unitarity from inelastic channels. In Section III we treat the inelastic case explicitly, and discuss the continuum ambiguity.

* Work supported in part by the National Science Foundation and a NATO Research Grant. 
In the domain of elastic unitarity, the nonlinear equation for the absorptive part $A(z)$ is

where

$$
A(z)=B(z ; A)
$$

$$
\begin{aligned}
B(z ; A) & =\sum_{l=0}^{\infty}(2 l+1)\left(A_{l}^{2}+D_{l}^{2}\right) P_{l}(z) \\
A_{l} & =\frac{1}{2} \int_{-1}^{1} d x P_{l}(x) A(x) \\
D_{l} & =\frac{1}{2} \int_{-1}^{1} d x P_{l}(x) D(x)
\end{aligned}
$$

and

$$
D(z)=\left[\sigma(z)-A^{2}(z)\right]^{\frac{1}{2}} .
$$

The unitary, analytic scattering amplitude is determined from a solution of Eq. (1.1) by

$$
f(z)=D(z)+i A(z) .
$$

As in Ref. [2], $\sigma(z)$ is a specified function which is analytic in $S\left(z_{0}\right)$, the interior of a unifocal ellipse of semi-major axis $z_{0}>1$. The Banach space of Ref. [2] was composed of real-analytic functions $A(z)$, which are analytic in $z$ in $S\left(\zeta_{1}\right)$, the interior of a unifocal ellipse of semi-major axis $\zeta_{1}$, where

$$
z_{0}<\zeta_{1}<2 z_{0}^{2}-1
$$

The norm was

$$
\|A\|=\sup _{l=0,1, \ldots}\left|A_{l}\right| / Q_{l}\left(\zeta_{1}\right)
$$

where $A_{l}$ are the partial-wave projections of $A(z)$, given by (1.3), and $Q_{l}$ are Legendre functions of the second kind.

Let $K_{b}$ be a certain finite ball of the Banach space, defined by $K_{b}=\{A:\|A\| \leqq b\}$. It was shown in Ref. [2] that, with suitable restrictions on $\sigma$, the functions $A$ in $K_{b}$ were mapped by (1.2)-(1.5) into functions $B$, all of which lie in a relatively compact subset of $K_{b}$. Schauder's fixed point theorem [3] was then used to establish the existence of at least one solution of Eq. (1.1) in the ball $K_{b}$.

The work of Ref. [2] has the shortcomings that (1) the ball radius $b$ approaches zero as $z_{0}$, the semi-major axis of the elliptical region $S\left(z_{0}\right)$, approaches 1, and (2) $b$ becomes small also as $\zeta_{1}$ is taken to be close to $2 z_{0}^{2}-1$. At asymptotically high energies the semi-major axis $z_{0}$ of the Lehmann ellipse, the ellipse of analyticity of the scattering amplitude, $f(z)$, is expected from general considerations to approach 1. In addition, if $\sigma(z)$ is analytic and bounded in $S\left(z_{0}\right)$, one similarly expects the absorptive part $A(z)$ to be analytic and bounded within an ellipse of semi-major 
axis equal to $2 z_{0}^{2}-1$. We will show here that both these shortcomings are not intrinsic to the nonlinear Eq. (1.2)-(1.5), but may be avoided by a more suitable Banach norm.

Here we choose a norm such that a finite ball of the Banach space is mapped into itself in the limit as $z_{0}$ approaches +1 . To motivate our choice of norm, let us take $z_{0}=1$ and consider the mapping of $A$ into $B$, given by (1.2)-(1.4), under the following restrictions:

(1) there exist constants $\kappa$ and $\mu$, with $5 / 2<\mu<3$, such that ${ }^{1}$

$$
\left|A_{l}\right| \leqq \kappa /(l+1)^{\mu},
$$

(2) $\sigma(z)$ is continuously differentiable for $-1 \leqq z \leqq+1$, and

(3) $D(z)$ has no zeros for $-1 \leqq z \leqq+1$. Under these conditions, one may justify an integration by parts ${ }^{2}$ of Eq. (1.4) and obtain that

$$
\left|D_{l}\right| \leqq \kappa /(l+1)^{\frac{3}{2}} .
$$

Consequently, we have that

$$
B_{l}=\left|D_{l}^{2}+A_{l}^{2}\right| \leqq \kappa /(l+1)^{3} \leqq \frac{\kappa}{(l+1)^{\mu}} .
$$

Thus the large-l bound of (1.9) is reproduced by the nonlinear mapping (1.2)-(1.4), with appropriate restrictions on $\sigma$. Our Banach norm (2.1) is chosen so that elements of the space satisfy (1.9) uniformly as $z_{0}$ approaches 1 . It is shown in Section II that a finite ball of the Banach space is mapped into itself by (1.2)-(1.4), and one can choose the ball radius $b$ to be independent of $z_{0}$.

In Appendix B we discuss a compactness criterion which is natural for subsets of Banach spaces with a discrete norm, such as our norm (2.1). We have not seen such a criterion used previously in the physics literature on nonlinear analysis. This compactness criterion enables us to take $z_{1}=2 z_{0}^{2}-1$ and still be able to map a finite ball through the system (this was not possible in Ref. [2]).

In Appendix A we use the Laplace representations of the Legendre functions $P_{l}(z)$ and $Q_{l}(z)$ to obtain certain upper bounds which are valid when $l$ is a non-negative integer and $z$ is complex. The bounds (A 21) and (A 34) on Legendre functions, along with bounds (2.32) and (2.36) on their derivatives, are used extensively in the analysis of the nonlinear mapping (1.2)-(1.4). These bounds are optimal at large $l$ for $z$ near \pm 1 . The argument is given in considerable detail, since the underlying technique and the bounds themselves may be of more general interest.

1 Throughout this section $k$ is a generic constant; it does not have the same value in different relations in which it appears.

2 The technique of integration by parts is discussed in Appendix C. The results obtained there reduce to (1.10) uniformly as $z_{0}$ approaches 1 . 
In Section III we consider a given unitary scattering amplitude $f(z)$, for a fixed energy above the inelastic threshold, which is analytic in $z$ in the elliptical region $S\left(z_{0}\right)$. We show that this amplitude is merely one of a continuum of unitary amplitudes analytic in $S\left(z_{0}\right)$, all of which correspond to the same cross-section $\sigma(z)$. This continuum of scattering amplitudes may be generated, as in Ref. [2], by varying the inhomogeneous contribution to unitarity, the function $I(z)$ defined by Eq. (3.2).

We shall require in Section III that the dispersive part of the given scattering amplitude, $D(z)$, does not vanish within $S\left(z_{0}\right)$. If $D(z)$ does have a zero inside $S\left(z_{0}\right)$, the inelasticities $\eta_{l}$ in $I(z)$ may not be varied arbitrarily. In fact, there must be one constraint on the variation of the $\eta_{l}$ for every (simple) zero of $D(z)$ inside $S\left(z_{0}\right)$. Thus, a continuum ambiguity in the determination of the amplitude $f(z)$ from the cross-section $\sigma(z)$ is present even if $D(z)$ has zeros inside $S$. We will not prove this result here; the details of the proof for this case are quite analogous to those explicitly discussed in Sections III and IV of Ref. [4].

Certain of the limitations of Ref. [2] were overcome in Ref. [4] by means of a technique which largely avoids estimates concerning Legendre functions. In particular, in the discussion of Ref. [4], the ball radius does not tend to zero as $z_{0}$ approaches 1 . Analyticity of the absorptive part in the large Lehmann ellipse $S\left(z_{1}\right)$ is obtained, but boundedness and differentiability on the boundary, which are obtained in the present work, are not guaranteed. The compactness criterion used in Ref. [4] works smoothly for open regions, but a more powerful (if more laborious) technique such as that used here seems to be required to handle the boundary. Control of behavior on the boundary is of some interest, since one might wish eventually to specify the singularity of $\sigma(z)$ on the boundary of its region of analyticity. This might be required in a phenomenological program in which one attempted to specify certain features of the interaction; i.e., a generalized "polology".

\section{Section II : Fixed Point Proof}

Here we will show that Eq. (1.1) has a solution $A(z)$ which is analytic in the open unifocal elliptical region $S\left(z_{1}\right)$, with semi-major axis $z_{1}=2 z_{0}^{2}-1^{3}$, if one has a cross-section $\sigma(z)$ analytic in $S\left(z_{0}\right)$ and subject to certain other restrictions to be given presently. To this end we define a Banach space $\mathscr{B}$ of real-analytic functions $h(z)$ analytic for $z \in S\left(z_{1}\right)$. We choose the norm to be

$$
\begin{aligned}
\|h\|= & \sup \left\{\left|h_{0}\right|, 2^{\mu}\left(z_{1}+\sqrt{z_{1}^{2}-1}\right)^{\frac{1}{2}}\left|h_{1}\right|,\right. \\
& \left.\sup _{l=2,3, \ldots}(l+1)^{\mu}\left(z_{1}+\sqrt{z_{1}^{2}-1}\right)^{l-1}\left|h_{l}\right|\right\}
\end{aligned}
$$

${ }^{3}$ With this choice, $z_{1}+\sqrt{z_{1}^{2}-1}=\left(z_{0}+\sqrt{z_{0}^{2}-1}\right)^{2}$. 
where $5 / 2<\mu<3$. The partial wave projections $h_{l}$ are real and are defined by

$$
h_{l}=\frac{1}{2} \int_{-1}^{1} d z P_{l}(z) h(z) .
$$

The elements $h(z)$ of the Banach space are analytic for $z \in S\left(z_{1}\right)$ because their Legendre series representation can be shown to be uniformly convergent for $z$ in $S\left(z_{1}\right)$. Uniform convergence follows easily from

$$
\begin{gathered}
\left|h_{0}\right| \leqq\|h\|, \\
\left|h_{1}\right| \leqq \frac{\|h\|}{2^{\mu}} \frac{1}{z_{0}+\sqrt{z_{0}^{2}-1}},
\end{gathered}
$$

and

$$
\left|h_{l}\right| \leqq \frac{\|h\|}{(l+1)^{\mu}} \cdot \frac{1}{\left(z_{1}+\sqrt{z_{1}^{2}-1}\right)^{l-1}}
$$

for $l \geqq 2$, when used along with the bound

$$
\left|P_{l}(z)\right| \leqq\left(z+\sqrt{z^{2}-1}\right)^{l},
$$

which is established in Appendix A.

Furthermore, we may use (2.3)-(2.6) to show that when $z$ is in the smaller ellipse $S\left(z_{0}\right)$, and $\|h\| \leqq b$, then $|h(z)|$ is bounded above by a constant independent of $z_{0}$ :

$$
\begin{aligned}
|h(z)| & \leqq \sum_{l=0}^{\infty}(2 l+1)\left|h_{l}\right|\left|P_{l}(z)\right| \\
& \leqq b\left\{1+\frac{3}{2^{\mu}}+\sum_{l=2}^{\infty} \frac{(2 l+1)}{(l+1)^{\mu}} \frac{\left|P_{l}(z)\right|}{\left(z_{1}+\sqrt{z_{1}^{2}-1}\right)^{l-1}}\right\} \\
& \leqq b\left\{1+\frac{3}{2^{\mu}}+\sum_{l=0}^{\infty} \frac{\left(2^{l}+1\right)}{(l+1)^{\mu}}\right\} \\
& \leqq 2 b \sum_{l=0}^{\infty} \frac{1}{(l+1)^{\mu-1}} \equiv \beta b .
\end{aligned}
$$

We will consider the mapping of $A(z) \in \mathscr{B}$ into $B(z)$ given by (1.2)-(1.4), with the cross-section $\sigma(z)$ analytic in $z$ and free of zeros for $z \in S\left(z_{0}\right)$. It will be established that, with appropriate restrictions on $\sigma(z)$, the operator maps $K_{b}$ into $a$ subset of $K_{b}$, where the set $K_{b}$ is defined to be the ball

$$
K_{b}=\{A \mid A \in \mathscr{B} \quad \text { and } \quad\|A\| \leqq b\},
$$

with $b$ to be chosen later. Furthermore, we will show that $B(A)$ is a continuous mapping in the topology induced by the norm (2.1). Finally, we 
will prove that the set of images $B\left(K_{b}\right)$ is a compact set in the topology induced by (2.1).

Thus, we shall be able to establish that $B(A)$ is a continuous mapping of a closed, convex set $K_{b}$ in the Banach space $\mathscr{B}$ into the compact subset $B\left(K_{b}\right)^{4}$. The conditions of Schauder's fixed point theorem are met [3], and we will be able to conclude that at least one solution of Eq. (1.1) exists and lies in the ball $K_{b}$.

For the work of this section we wish to constrain the ball $K_{b}$ so that

$$
|A(z)|^{2}<|\sigma(z)|
$$

for $A \in K_{b}$ and $z$ in the closed elliptical region $\bar{S}\left(z_{0}\right)$. If we use the bound (2.7), which is satisfied by any element of $K_{b}$, for $|A(z)|$, we see that (2.9) is satisfied if

$$
(\beta b)^{2}<m^{2},
$$

where we make the definition ${ }^{5}$

$$
m^{2}=\inf _{z \in \hat{\imath} S\left(z_{0}\right)}|\sigma(z)| .
$$

We also require that $|\sigma(z)|$ and $\left|\sigma^{\prime}(z)\right|$ have finite maximum values on $\partial S\left(z_{0}\right)$, which are given by

$$
\begin{aligned}
& M^{2}=\sup _{z \in \hat{\Gamma} S\left(z_{0}\right)}|\sigma(z)|, \\
& 2 N=\sup _{z \in \hat{\Gamma} S\left(z_{0}\right)}\left|\sigma^{\prime}(z)\right| .
\end{aligned}
$$

If condition (2.10) is met, the function $D(z)$, defined by (1.5), is analytic for $z$ in the ellipse $S\left(z_{0}\right)$. Also, $D(z)$ is continuous and bounded on the boundary $\partial S\left(z_{0}\right)$, and it may be represented for physical $z$ by a Cauchy integral over $\partial S\left(z_{0}\right)$. We may use this Cauchy integral, along with the relation

$$
Q_{l}(z)=\frac{1}{2} \int_{-1}^{1} d x \frac{P_{l}(x)}{z-x}
$$

to write the partial wave projections of $D$ as

$$
D_{l}=\frac{1}{2 \pi i} \int_{i S\left(z_{0}\right)} d z Q_{l}(z) D(z) .
$$

In order to show that a certain ball $K_{b} \subset \mathscr{B}$ is mapped into a compact subset $B\left(K_{b}\right)$ by (1.2)-(1.4), we will need bounds upon $B_{l}$, the partial

${ }^{4}$ Convexity of the ball $K_{b}$ follows from linearity of the norm (2.1).

${ }^{5}$ Since $\sigma(z)$ is free of zeros in the closed ellipse $\bar{S}\left(z_{0}\right)$, it takes its minimum modulus over $\bar{S}\left(z_{0}\right)$ on the boundary, $\lambda S\left(z_{0}\right)$. 
waves of $B(z)$, which are given by

$$
B_{l}=D_{l}^{2}+A_{l}^{2} .
$$

To establish that $B\left(K_{b}\right) \subset K_{b}$, we must show that for all $A(z) \in K_{b}$,

$$
\begin{gathered}
\left|B_{0}\right| \leqq b, \\
\left|B_{1}\right| \leqq \frac{b}{2^{\mu}} \cdot \frac{1}{z_{0}+\sqrt{z_{0}^{2}-1}},
\end{gathered}
$$

and

$$
\left|B_{l}\right| \leqq \frac{b}{(l+1)^{\mu}}\left[z_{1}+\sqrt{z_{1}^{2}-1}-(l-1)\right.
$$

for $l=2,3, \ldots$ Furthermore, $B\left(K_{b}\right)$ will be compact in the topology induced by the norm (2.1) if, for any number $\varepsilon>0$, there exists an integer $L(\varepsilon)$ such that

$$
\sup _{l \geqq L}(l+1)^{\mu}\left[z_{1}+\sqrt{z_{1}^{2}-1}\right]^{l-1}\left|B_{l}\right| \leqq \varepsilon
$$

for every element $A$ of $K_{b}$. This compactness criterion (2.20) is justified in Appendix $\mathrm{B}^{6}$.

Clearly, if $b$ is chosen sufficiently small, the term $A_{l}^{2}$ in (2.16) is appropriate for establishing (2.17)-(2.20). Hence we need only to obtain appropriate bounds on the $D_{l}^{2}$ term. Let us first consider $l=0$. From (1.5), (2.7), and (2.12) we obtain for $z \in S\left(z_{0}\right)$ that

$$
|D(z)| \leqq\left[M^{2}+\beta^{2} b^{2}\right]^{\frac{1}{2}} .
$$

This bound may be used in (1.4) to obtain

$$
\left|D_{0}\right| \leqq\left[M^{2}+\beta^{2} b^{2}\right]^{\frac{1}{2}} \text {. }
$$

For $l=1$, let us use the Laplace representation for $Q_{1}(z)$, Eq. (A 20) of Appendix A, to obtain for $z \in \partial S\left(z_{0}\right)$ that

$$
\begin{aligned}
\left|Q_{1}(z)\right| & \leqq \frac{1}{z_{0}+\sqrt{z_{0}^{2}-1}} \int_{0}^{\infty} \frac{d u}{\mid \sqrt{z^{2}-1 \mid \cosh u}} \\
& =\frac{\pi}{2} \frac{1}{z_{0}+\sqrt{z_{0}^{2}-1}} \frac{1}{\mid \sqrt{z^{2}-1 \mid}} .
\end{aligned}
$$

${ }^{6}$ This criterion, in contrast to the treatments of Refs. [2] and [6], does not require that $B(z)$ be analytic in a domain larger than that assumed for $A$. Crudely speaking, it requires that the derivative of $B(z)$ be Hölder continuous in $z$ with a larger Hölder exponent than the elements $A(z)$ of $K_{b}$. The compactness criterion (2.23) is more appropriate for Banach spaces of sequences, and it allows us to carry through the proof for $z_{1}$ equal to $2 z_{0}^{2}-1$. 
We use (2.15), (2.21), and (2.23) to obtain

$$
\left|D_{1}\right| \leqq\left[M^{2}+\beta^{2} b^{2}\right]^{\frac{1}{2}} \frac{1}{4} \frac{1}{z_{0}+\sqrt{z_{0}^{2}-1}} \cdot \int_{\partial S\left(z_{0}\right)} \frac{d z}{\left|z^{2}-1\right|^{\frac{1}{2}}} .
$$

One can show that

$$
\int_{\partial S\left(z_{0}\right)} \frac{|d z|}{\left|z^{2}-1\right|^{\frac{1}{2}}}=2 \pi
$$

[The proof of (2.25) is analogous to that of bounds on (2.37) and (2.38) ] We obtain a bound on $\left|D_{1}\right|$ of the form:

$$
\left|D_{1}\right| \leqq \frac{\pi}{2} \frac{\left[M^{2}+\beta^{2} b^{2}\right]^{\frac{1}{2}}}{z_{0}+\sqrt{z_{0}^{2}-1}} .
$$

To obtain appropriate bounds on $D_{l}$ for $l \geqq 2$ we integrate (2.15) by parts to obtain

$$
D_{l}=\frac{1}{l(l+1)} \frac{1}{2 \pi i} \int_{i S\left(z_{0}\right)} d z\left(1-z^{2}\right) Q_{l}^{\prime}(z) D^{\prime}(z) .
$$

This result is justified in Appendix $C$. We use (1.5) to express the derivative of $D$ in terms of $A$ and $\sigma$ :

$$
D^{\prime}(z)=\left\{\frac{\sigma^{\prime}(z)}{2}-A(z) A^{\prime}(z)\right\} / D(z) .
$$

We have required via (2.10) that $|D(z)|$ be bounded below; let us define

$$
n=\inf _{z \in S\left(z_{0}\right)}|D(z)| \text {. }
$$

We use (2.7), (2.13), and (2.29) to bound $D^{\prime}$ :

$$
\left|D^{\prime}(z)\right| \leqq\left[N+\beta b\left|A^{\prime}(z)\right|\right] / n .
$$

We use (2.30) in conjunction with an upper bound on $\left|A^{\prime}(z)\right|$ for $z \in \partial S\left(z_{0}\right)$, to bound the integral in (2.27). The upper bound on $\left|A^{\prime}\right|$ is obtained as follows: First, differentiate the Legendre series for $A$ term by term and use (2.3)-(2.5) to obtain

$$
\left|A^{\prime}(z)\right| \leqq b\left\{\frac{3}{2^{\mu}}+\sum_{l=2}^{\infty} \frac{(2 l+1)}{(l+1)^{\mu}} \cdot\left[z_{1}+\sqrt{z_{1}^{2}-1}\right]^{-(l-1)}\left|P_{l}^{\prime}(z)\right|\right\} .
$$

In Appendix A we obtain the following bound on $\left|P_{l}^{\prime}\right|$ :

$$
\left|P_{l}^{\prime}(z)\right| \leqq \frac{2 \sqrt{2}}{\sqrt{\pi}} \frac{1}{\left|z^{2}-1\right|^{\frac{3}{4}}}\left(z_{0}+\sqrt{z_{0}^{2}-1}\right)^{l+\frac{1}{2}} \cdot \frac{l+1}{\sqrt{l}} .
$$


We then use (2.32) in (2.31) to obtain

where

$$
\left|A^{\prime}(z)\right| \leqq \frac{3 b}{2^{\mu}}+\frac{\gamma b}{\left|z^{2}-1\right|^{\frac{3}{4}}},
$$

$$
\gamma=\frac{4 \sqrt{2}}{\sqrt{\pi}} \sum_{l=2}^{\infty} \frac{1}{(l+1)^{\mu-2} l^{\frac{1}{2}}} .
$$

We use (2.33) and (2.30) in (2.27) to obtain

$$
\begin{aligned}
\left|D_{l}\right| \leqq \frac{1}{l(l+1)} \cdot \frac{1}{2 \pi n} \int_{\partial S\left(z_{0}\right)}|d z|\left|z^{2}-1\right|\left|Q_{l}^{\prime}(z)\right| \\
\cdot\left\{N+\frac{3 \beta}{2^{\mu}} b^{2}+\beta \gamma b^{2} \frac{1}{\left|z^{2}-1\right|^{\frac{3}{4}}}\right\} .
\end{aligned}
$$

We majorize the integral (2.35) by using the bound

$$
\left|Q_{l}^{\prime}(z)\right| \leqq \frac{10 \sqrt{l+1}}{\left|z^{2}-1\right|}\left\{z_{0}+\sqrt{z_{0}^{2}-1}\right\}^{-l}
$$

for $z \in \partial S\left(z_{0}\right)$, which is also obtained in Appendix A. We obtain an estimate of $\left|D_{l}\right|$ from (2.35) and bounds on the integrals

and

$$
\begin{aligned}
& I=\int_{i S\left(z_{0}\right)}|d z| \frac{1}{\left|z^{2}-1\right|^{\frac{3}{4}}} \\
& J=\int_{i S\left(z_{0}\right)}|d z| \leqq 2 \pi z_{0} .
\end{aligned}
$$

We may change the integration variable in (2.37) to $\phi$, where $z=z_{0} \cos \phi$ $+i \sqrt{z_{0}^{2}-1} \sin \phi$, obtaining

$$
I=\int_{0}^{2 \pi} d \phi\left\{z_{0}^{2} \sin \phi+\left(z_{0}^{2}-1\right) \cos ^{2} \phi\right\}^{-\frac{1}{4}}<\int_{0}^{2 \pi} d \phi|\sin \phi|^{-\frac{1}{2}}<4 \pi .
$$

The resulting bound for $\left|D_{l}\right|$ is

$$
\left|D_{l}\right| \leqq \frac{10}{l \sqrt{l+1}} \frac{1}{n}\left\{z_{0}+\sqrt{z_{0}^{2}-1}\right\}^{-l}\left\{z_{0}\left(N+\frac{3 \beta}{2^{\mu}} b^{2}\right)+2 \beta \gamma b^{2}\right\} \text {. }
$$

As a consequence we obtain

$$
\begin{aligned}
& \left|D_{l}\right|^{2}(l+1)^{\mu}\left(z_{1}+\sqrt{z_{1}^{2}-1}\right)^{l-1} \\
& \quad \leqq \frac{100}{n^{2}} \cdot \frac{(l+1)^{\mu-1}}{l^{2}}\left[N+b^{2} \beta\left(\frac{3}{4}+2 \gamma\right)\right]^{2} .
\end{aligned}
$$

The right sides of (2.22), (2.26), and (2.41) are independent of $z_{0}$. 
We will now establish that $B\left(K_{b}\right) \subset K_{b}$ for an appropriate choice of $b$. Conditions (2.17) and (2.18) are met if

$$
b^{2}+20\left(M^{2}+\beta^{2} b^{2}\right) \leqq b,
$$

and (2.19) is met if

$$
b^{2}+\frac{100}{n^{2}} \frac{(l+1)^{\mu-1}}{l^{2}}\left[N+b^{2} \beta\left(\frac{3}{4}+2 \gamma\right)\right]^{2} \leqq b .
$$

We must also satisfy (2.10), so that $D(z)$ will not vanish on $\bar{S}\left(z_{0}\right)$. Let us require that

$$
b \leqq \frac{m}{\sqrt{2} \beta},
$$

so that

$$
n^{2} \geqq \frac{m^{2}}{2} .
$$

We may ensure that (2.42) and (2.43) are satisfied if (2.44) is met and if ${ }^{7}$

where

$$
b^{2}+L \leqq b,
$$

$$
L=\max \left\{10\left(2 M^{2}+m^{2}\right), \frac{450}{m^{2}}\left[N+\frac{m^{2}}{\beta}\left(\gamma+\frac{3}{8}\right)\right]^{2}\right\} .
$$

Let us require that

$$
4 L<1 ;
$$

this condition will be met if, for example, $\sigma(z)$ is nearly constant and sufficiently small. Under this condition, (2.46) is satisfied for

where

$$
b_{-} \leqq b \leqq b_{+} \text {, }
$$

$$
b_{+}=\frac{1}{2}\left\{1 \pm[1-4 L]^{\frac{1}{2}}\right\} .
$$

Finally, we note that (2.42), (2.43), and (2.44) are satisfied for $b$ slightly greater than $b_{-}$, if condition (2.48) is met and if

$$
b_{-} \cdot \frac{\sqrt{2} \beta}{m}<1 \text {. }
$$

Again, this condition can be satisfied if $\sigma(z)$ is small and slowly varying. The constraints (2.48) and (2.51) are sufficient to guarantee that $B\left(K_{b}\right) \subset K_{b}$.

Conditions (2.48) and (2.51) guarantee also that the mapping of $A(z)$ into $B(z)$, given by (1.2)-(1.4), is continuous in the topology induced by

\footnotetext{
${ }^{7}$ In condition (2.44), we have used the relation $(l+1)^{\mu-1} / l^{2} \leqq 9 / 4$, which is valid for $\mu<3$ and $l \geqq 2$.
} 
(2.1). To establish this, we first show that, for $A_{1}$ and $A_{2}$ in $K_{b}$, $\left|D_{1}^{\prime}(z)-D_{2}^{\prime}(z)\right|$ is small on the boundary $\partial S\left(z_{0}\right)$ when $A_{1}$ is close in norm to $A_{2}$. It then follows from a straightforward analysis of (2.27) that $l^{\frac{3}{2}}\left(z_{0}+\sqrt{z_{0}^{2}-1}\right)^{l-1}\left|D_{1 l}-D_{2 l}\right|$ is also small. Finally, we establish via (2.16) that $l^{3}\left(z_{1}+\sqrt{z_{1}^{2}-1}\right)^{l-1}\left|B_{1 l}-B_{2 l}\right|$ is small. The separate analysis for $l=0$ and 1 is trivial, so that we may conclude that $\left\|B_{1}-B_{2}\right\|$ vanishes as $\left\|A_{1}-A_{2}\right\|$ tends to zero. Continuity of the mapping is thus established.

We establish compactness of the image set $B\left(K_{b}\right)$ via the criterion (2.20). This inequality is easily established, however, since from (2.40) and (2.16) we may conclude that $\left|B_{l}\right|$ is of asymptotic order

$$
\left\{z_{1}+\sqrt{z_{1}^{2}-1}\right\}^{-(l-1)} / l^{3}
$$

at large $l$ and since we assume $\mu<3$, we may ensure (2.20) by an appropriately large choice of $L(\varepsilon)$.

The restrictions (2.48) and (2.51) are thus sufficient to guarantee that the hypotheses of Schauder's theorem are met, so that there is at least one solution of (1.1)-(1.4) in the ball $K_{b}$.

\section{Section III: Continuum Ambiguity}

Here we assume the existence of a particular scattering amplitude at a fixed energy, which satisfies unitarity with a certain inelastic contribution and which corresponds to the cross-section $\sigma(z)$. Let us decompose this scattering amplitude, $f_{0}(z)$, into its dispersive part $D_{0}(z)$ and its absorptive part $A_{0}(z)$. We shall assume that $\sigma(z)$ is analytic in the ellipse $S\left(z_{0}\right)$, with a bounded derivative on the boundary, $\partial S\left(z_{0}\right)$. We require that $A_{0}(z)$ be in the Banach space $\mathscr{B}$ of functions which are analytic inside the larger ellipse $S\left(z_{1}\right)$. Also, we wish to require that $D_{0}(z)$, which is analytic and bounded in the region $S\left(z_{0}\right)$, have no zeros in its closure, $\bar{S}\left(z_{0}\right)$. We will show that, under weaker conditions, one may construct a continuum of unitary scattering amplitudes, all with the same cross-section $\sigma$, by varying the inelastic contribution to unitarity. Thus we establish the existence of a continuum of acceptable unitary amplitudes, all giving the same cross-section.

The equation to be satisfied by the imaginary part in the inelastic regime is

$$
A(z)=P(z ; A)=B(z ; A)+I(z),
$$

where $B(z ; A)$ is given by Eqs. (1.2)-(1.4), and the inelastic contribution is

with

$$
I(z)=\sum_{l=0}^{\infty}(2 l+1) I_{l} P_{l}(z),
$$

$$
I_{l}=\frac{1}{4}\left(1-\eta_{l}^{2}\right) \text {. }
$$


Let us require that $I(z)$ lie in the Banach space $\mathscr{B}$, so that for $I(z)$ the norm (2.1) is finite. Let us also require

$$
0 \leqq \eta_{l} \leqq 1
$$

so that inelastic unitarity is valid for the scattering amplitude $f=D+i A$, where $A$ satisfies (3.1) and $D$ is given by (1.5).

It is a consequence of the analysis of Section II, and the requirement that $I$ be in $\mathscr{B}$, that $P(z ; A)$ belongs to $\mathscr{B}$ when $A$ lies in a sufficiently small neighborhood of $A_{0}$. Let us note from (3.1) that the partial waves of $P$ are

$$
P_{l}=B_{l}+I_{l} .
$$

Let the function $I(z)$ depend upon a parameter $\lambda$ (which is a prescribed function of the elasticities), such that (3.4) is satisfied and $\|I(z ; \lambda)\|$ is finite for $\lambda$ in some neighborhood of the initial values, $\lambda_{0}$. We wish to establish the conditions necessary to apply an appropriate implicit function theorem to guarantee the existence of a solution of (3.1), $A(z, \lambda)$, for $\lambda$ in some neighborhood of $\lambda_{0}$. We will require that the derivative of the inhomogeneous term $I(z, \lambda)$ with respect to $\lambda$ be in the Banach space $\mathscr{B}$, and depend continuously on $\lambda$.

As a prelude to the implicit function argument, we must show that $P_{A}\left(A_{0}\right) h$, the Frechét derivative of $P$ with respect to the function $A$ evaluated at $A_{0}$ and applied to an arbitrary element $h$ of $\mathscr{B}$, has certain properties.

Since uniform convergence is guaranteed for the relevant series and integrals for $A$ sufficiently close to $A_{0}$ with $h \in \mathscr{B}$, one may write $P_{A}(A) \cdot h$ as

with

$$
p(z) \equiv P_{A}(A) h(z)=\sum_{l=0}^{\infty}(2 l+1) P_{l}(z) p_{l}
$$

$$
\begin{aligned}
p_{l} & =\int_{-1}^{1} d x P_{l}(x) h(x)\left\{A_{l}-D_{l} A(x)\left[\sigma(x)-A^{2}(x)\right]^{-\frac{1}{2}}\right\} \\
& =2\left[A_{l} h_{l}-D_{l} q_{l}\right],
\end{aligned}
$$

where $h_{l}$ are the partial-wave projections of $h(z)$, and $q_{l}$ are the partialwave projections of

$$
q(z)=h(z) A(z) / D(z) \text {. }
$$

In order to apply the implicit function theorem to (3.1), we establish the following properties of $P_{A}(A)$ :

(1) The formal expression (3.6) for $P_{A}(A)$ is in fact the Frechét derivative of $P$ on $\mathscr{B}$, which exists for $A$ in a neighborhood of $A_{0}$.

(2) $P_{A}(A)$ is continuous in $A$ at $A_{0}$.

(3) $P_{A}\left(A_{0}\right)$ is a completely continuous linear operator on $\mathscr{B}$. 
One establishes these properties for $P_{A}$ in a manner quite analogous to the analysis of $B(z ; A)$ in Section II. In particular, note that, like the function $D(z)$ of Section II, $q(z)$ is analytic in $S\left(z_{0}\right)$, and its derivative on the boundary $\partial S\left(z_{0}\right)$ is bounded. One may obtain bounds upon the partial waves $q_{l}$ of the same form as relation (2.40) for the partial waves of $D$. This estimate, along with subsidiary estimates for $l=0$, and appropriate bounds on $\left|A_{l}\right|,\left|h_{l}\right|$, and $\left|D_{l}\right|$, allows us to establish these properties. For (3) we apply the compactness criterion discussed in Appendix B. The argument is similar to our proof of compactness of the set $B\left(K_{b}\right)$ in Section II.

We are considering Eq. (3.1), which we write in the form

$$
F(z ; A, \lambda)=A(z ; \lambda)-P(z ; A)=0 .
$$

We are given a solution $A_{0}(z)$, corresponding to $\lambda=\lambda_{0}$. We will establish the existence of a solution $A(z ; \lambda)$ of (3.9) for $\lambda$ in some neighborhood of $\lambda_{0}$.

Let us make the additional assumption that the homogeneous equation

$$
F_{A}\left(A_{0}\right) \psi=0
$$

has no nontrivial solutions. This condition does not follow from the previous assumptions, and we shall not discuss the exceptional case in which it is not met. If the condition is met, the operator $F_{A}(A)$ has an inverse on $\mathscr{B}$ [5]. Furthermore, we can use an appropriate form of the implicit function theorem ${ }^{8}$ to guarantee the existence of a solution to (3.1), $A(z, \lambda)$, in the Banach space $\mathscr{B}$. One may generate $A(z ; \lambda)$ from $A\left(z, \lambda_{0}\right)=A_{0}(z)$ by solving the differential equation

$$
\frac{d A}{d \lambda}(z, \lambda)=-\left[F_{A}(A, \lambda)\right]^{-1} F_{\lambda}(z ; A, \lambda) .
$$

We have established a continuum ambiguity in the determination of the scattering amplitude from the cross-section via unitarity, in a Banach space $\mathscr{B}$ of analytic functions. Considerations have been limited to the case for which $\sigma-A^{2}$ does not vanish in the closed elliptical region $\bar{S}\left(z_{0}\right)$. An $)$ zeros of $\sigma-A^{2}$ in $\bar{S}\left(z_{0}\right)$ do change the nature of this continuum ambiguity, but do not remove the ambiguity. In fact, it can be shown here, as it was shown under similar circumstances in Ref. [4], that for each simple zero of $\sigma-A^{2}$ within $S\left(z_{0}\right)$ c one must place one constraint upon the variation of the elasticities $I_{l}$ with respect to the parameter $\lambda$.

\footnotetext{
${ }^{8}$ For a discussion, see Ref. [3], pp. $554-561$.
} 


\section{Appendix A}

Here we will obtain bound (2.6) for $P_{l}(z),(2.32)$ for $P_{l}^{\prime}(z)$, and (2.36) for $Q_{l}^{\prime}(z)$, where $l$ is an integer and $z$ is a point on the conventional "physical" sheet of the complex plane. We can consider the point $z$ to be on a unifocal ellipse of semi-major axis $z_{0} \geqq 1$ :

$$
z=z_{0} \cos \phi+i \sqrt{z_{0}^{2}-1} \sin \phi, \quad(0 \leqq \phi \leqq 2 \pi) .
$$

We define $\sqrt{z^{2}-1}$ with the cut from -1 to +1 , so that

$$
\sqrt{z^{2}-1}=\sqrt{z_{0}^{2}-1} \cos \phi+i z_{0} \sin \phi \text {. }
$$

Consequently,

$$
z+\sqrt{z^{2}-1}=\left(z_{0}+\sqrt{z_{0}^{2}-1}\right) e^{i \phi} .
$$

The Laplace representation of $P_{l}(z)$ is

$$
P_{l}(z)=\frac{1}{\pi} \int_{0}^{\pi} d \omega\left\{z+\sqrt{z^{2}-1} \cos \omega\right\}^{l} .
$$

From (A 1) and (A 2) it follows that the phase difference of $z$ and $\sqrt{z^{2}-1}$ is less than or equal to $90^{\circ}$, so that

$$
\left|z+\sqrt{z^{2}-1} \cos \omega\right| \leqq\left|z+\sqrt{z^{2}-1}\right|=z_{0}+\sqrt{z_{0}^{2}-1},
$$

so that

$$
\left|P_{l}(z)\right| \leqq\left(z_{0}+\sqrt{z_{0}^{2}-1}\right)^{l} .
$$

Remark. We may use the Laplace representation to obtain

$$
\begin{aligned}
P_{l}^{\prime}(z)=\frac{l}{\pi} \int_{0}^{\pi} d \omega\{(z & \left.+\sqrt{z^{2}-1} \cos \omega\right)^{l-1} \\
& \left.+(l-1) z \sin ^{2} \omega\left(z+\sqrt{z^{2}-1} \cos \omega\right)^{l-2}\right\} .
\end{aligned}
$$

It follows immediately that

$$
\left|P_{l}^{\prime}(z)\right| \leqq \frac{l(l+1)}{2}\left(z_{0}+\sqrt{z_{0}^{2}-1}\right)^{l-1} .
$$

In order to motivate the bound (2.32) on $P_{l}^{\prime}(z)$ let us first obtain a corresponding bound upon $P_{l}(z)$ itself. We remove a factor of $\left(z+\sqrt{z^{2}-1}\right)^{l}$ and change the variable of integration to $v=1-\cos \omega$ to have

where

$$
A(y(z))=\left\{z+\sqrt{z^{2}-1}\right\}^{-l} P_{l}(z)=\frac{1}{\pi} \int_{0}^{2} \frac{d v}{\sqrt{v(2-v)}}\{1-y v\}^{l},
$$

$$
y=\frac{\sqrt{z^{2}-1}}{z+\sqrt{z^{2}-1}} .
$$


(A 1) and (A 2) imply that

$$
\left|y-\frac{1}{2}\right| \leqq \frac{1}{2}
$$

In particular, $\operatorname{Re} y \geqq 0$, with $\operatorname{Re} y=0$ only at $z= \pm 1$. Let us express $y$ in polar form:

$$
y=|y| e^{-i \theta}, \quad|\theta| \leqq \pi / 2 .
$$

It follows from (A9) that at a given phase $\theta,|y| \leqq \cos \theta$. We will obtain bounds explicitly for $0 \leqq \theta \leqq \pi / 2$; the bounds for negative $\theta$ will follow immediately from real analyticity of $A(y)$.

In order to obtain bounds that are uniform in $\theta$ we distort the integration over $v$ in (A 7) to be a "house top" contour with slope $\varrho=\theta / 2$; i.e., two line segments with endpoints $\left(0, e^{i \varrho} / \cos \varrho\right)$ and $\left(e^{i \varrho} / \cos \varrho, 2\right)$ :

$$
\begin{aligned}
& A=\frac{1}{\pi}\left(A_{1}+A_{2}\right) \\
& A_{1}=\int_{0}^{e^{i e} / \cos \varrho} \frac{d v}{\sqrt{v(2-v)}}\left\{1-v|y| e^{-i \theta}\right\}^{l}, \\
& A_{2}=\int_{0}^{e^{-i e / \cos e}} \frac{d v}{\sqrt{v(2-v)}}\left\{1-(2-v)|y| e^{-i \theta}\right\}^{l} .
\end{aligned}
$$

Note: $|2-v| \geqq 1$ in $A_{1}$ and $A_{2}$.

Let us change the variables of integration in $A_{1}$ and $A_{2}$ to $x=v e^{-i \varrho}$ and $v e^{i e}$, respectively:

$$
\begin{aligned}
& \left|A_{1}\right| \leqq \int_{0}^{1 / \cos \varrho} \frac{d x}{\sqrt{x}}|1-x| y\left|e^{-i \theta / 2}\right| l \\
& \left|A_{2}\right|=\int_{0}^{1 / \cos \varrho} \frac{d x}{\sqrt{x}}|1-| y\left|e^{-i \theta}\left(2-x e^{-i \theta / 2}\right)\right|^{l} .
\end{aligned}
$$

We define

$$
\begin{aligned}
& D(x)=1-x|y| e^{-i \theta / 2}, \\
& E(x)=1+x|y| e^{-3 i \theta / 2}-2|y| e^{-i \theta},
\end{aligned}
$$

for $0 \leqq|y| \leqq \cos \theta ; 0 \leqq x \leqq 1 / \cos \theta / 2$. We will obtain the following upper bonds on $|D|$ and $|E|$ :

$$
\begin{gathered}
\mathscr{D}(x,|y|)=|D|^{2}-1+x|y| \leqq 0, \\
\mathscr{E}(x,|y|)=|E|^{2}-1+x|y| \leqq 0 .
\end{gathered}
$$

$\mathscr{D}$ and $\mathscr{E}$ are second degree polynomials in $x$ and $|y|$; furthermore, the coefficients of $x^{2}$ and $|y|^{2}$ are positive, so that $\mathscr{D}$ and $\mathscr{E}$ take on their 
maximum values at one of the 4 points $(x,|y|)=(0,0),(0, \cos \theta)$, $\left(\frac{1}{\cos \theta / 2}, 0\right),\left(\frac{1}{\cos \theta / 2}, \cos \theta\right)$.

Let us consider $\mathscr{D}$ first. (A 14) is trivially true if either $x$ or $|y|$ is zero. Furthermore,

$$
\mathscr{D}(1 / \cos \theta / 2, \cos \theta)=\frac{\cos \theta}{\cos ^{2} \theta / 2}\{\cos \theta / 2-1\} \leqq 0 .
$$

So (A 14) is established.

As for $\mathscr{E},($ A 15$)$ is trivially true for $|y|=0$. In addition,

$$
\mathscr{E}(0,|y|)=|1-2 y|^{2}-1 \leqq 0
$$

by virtue of (A9), and

$$
\mathscr{E}(1 / \cos \theta / 2, \cos \theta)=\frac{\cos \theta}{\cos \theta / 2}\left\{1-\frac{1}{\cos \theta / 2}\right\} \leqq 0 .
$$

Furthermore, we may use the inequality $1-p \leqq e^{-p}$ for $0 \leqq p \leqq 1$ to obtain from (A 14) and (A 15) the bounds

$$
|D(x)| \leqq e^{-\frac{x|y|}{2}} \text { and }|E(x)| \leqq e^{-\frac{x|y|}{2}} .
$$

Whence, from (A 12) and (A 13),

$$
|A(y)| \leqq \frac{2}{\pi} \int_{0}^{1 / \cos \varrho} \frac{d x}{\sqrt{x}} e^{-\frac{l}{2} x|y|} .
$$

We may extend the integration out to infinity to obtain the bound

or

$$
|A(y)| \leqq 2\left(\frac{2}{\pi l|y|}\right)^{\frac{1}{2}}
$$

$$
\left|P_{l}(z)\right| \leqq 2\left(\frac{2}{\pi l}\right)^{\frac{1}{2}} \frac{1}{\left|z^{2}-1\right|^{\frac{1}{4}}}\left[z_{0}+\sqrt{z_{0}^{2}-1}\right]^{l+\frac{1}{2}} .
$$

We now obtain the bound (2.32) on $P_{l}^{\prime}$ by similar prestidigitation. From the Laplace integral (A4) one can obtain

$$
P_{l}^{\prime}(z)=\frac{l+1}{\pi} \frac{1}{\sqrt{z^{2}-1}} \cdot \int_{0}^{\pi} d \omega \cos \omega\left\{z+\sqrt{z^{2}-1} \cos \omega\right\}^{l} .
$$

Let us define $B(y)$ through the relation

$$
B(y(z))=\left[z+\sqrt{z^{2}-1}\right]^{-l} \frac{\sqrt{z^{2}-1}}{l+1} P_{l}^{\prime}(z) .
$$


We may again let $v=1-\cos \omega$ be the variable of integration and obtain

$$
B(y)=\frac{1}{\pi} \int_{0}^{2} \frac{d v}{\sqrt{v(2-v)}}(1-v)\{1-y v\}^{l}
$$

where $y$ is given in (A 8). We again distort the $v$ integration contour to a "house top" and decompose $B(y)$ as was done in (A11) with $A(y)$. After the contour distortion

$$
|1-v|=\left|1-x e^{\mp i \varphi}\right| \leqq 1
$$

for $0<\varrho<\pi / 4$ and $x$ in the domain of integration; hence we obtain analogously to (A21) the bound

$$
|B(y)| \leqq 2\left(\frac{2}{\pi l|y|}\right)^{\frac{1}{2}}
$$

and hence for $P_{l}^{\prime}$ the bound

$$
\left|P_{l}^{\prime}(z)\right| \leqq 2(l+1)\left(\frac{2}{\pi l}\right)^{\frac{1}{2}} \frac{1}{\left|z^{2}-1\right|^{\frac{3}{4}}}\left[z_{0}+\sqrt{z_{0}^{2}-1}\right]^{l+\frac{1}{2}} .
$$

Now we will obtain the bound (2.36) on $Q_{l}^{\prime}$ and an analogous bound upon $Q_{l}$ itself. The Laplace representation for $Q_{l}(z)$ is

$$
Q_{l}(z)=\int_{0}^{\infty} d u\left\{z+\sqrt{z^{2}-1} \cosh u\right\}^{-(l+1)}
$$

We define $C(y(z))=\left\{z+\sqrt{z^{2}-1}\right\}^{l+1} Q_{l}(z)$, with $y$ given in (A 8), to obtain

$$
C(y)=\int_{0}^{\infty} d u\{1+y(\cosh u-1)\}^{-(l+1)} .
$$

We change the integration variable to $x=\cosh u-1$ and obtain

$$
C(y)=\int_{0}^{\infty} \frac{d x}{\sqrt{x(x+2)}}\left\{1+|y| e^{-i \theta} x\right\}^{-(l+1)} .
$$

Then we rotate the $x$-contour through an angle $\theta$ :

$$
C(y)=\int_{0}^{\infty} \frac{d t}{\sqrt{t\left(t+2 e^{-i \theta}\right)}}\{1+|y| t\}^{-(l+1)} .
$$

Now we divide the integral (A 29) into two parts.

$$
\begin{aligned}
C(y) & =C_{1}+C_{2}, \\
C_{1} & =\int_{0}^{1 /|y|} \frac{d t}{\sqrt{t\left(t+2 e^{-i \theta}\right)}}\{1+|y| t\}^{-(l+1)}, \\
C_{2} & =\int_{1 /|y|}^{\infty} \frac{d t}{\sqrt{t\left(t+2 e^{-i \theta}\right)}}\{1+|y| t\}^{-(l+1)} .
\end{aligned}
$$


In the integral for $C_{1}$ we have $\left|t+2 e^{-i \theta}\right| \geqq 2$. Also, we use the inequality

$$
\frac{1}{1+p} \leqq e^{-p / 2}
$$

if $0 \leqq p \leqq 1$ to obtain

$$
\left|C_{1}\right| \leqq \int_{0}^{1 /|y|} \frac{d t}{\sqrt{2 t}} e^{-\frac{(l+1)|y| t}{2}}
$$

We may take the integration out to infinity to obtain

$$
\left|C_{1}\right| \leqq \frac{\sqrt{\pi}}{\sqrt{(l+1)|y|}}
$$

In the integral for $C_{2}$ we may use $\left|t+2 e^{-i \theta}\right| \geqq t$ to obtain

$$
\begin{aligned}
\left|C_{2}\right| & \leqq \int_{1 /|y|}^{\infty} \frac{d t}{t}\{1+|y| t\}^{-(l+1)} \leqq 2 \int_{0}^{\infty} d u\{1+u\}^{-(l+2)} \\
& =\frac{1}{(l+1)} \cdot \frac{1}{2^{l}} \leqq \frac{1}{|\sqrt{y}| \sqrt{l+1}}
\end{aligned}
$$

As a consequence,

$$
\left|Q_{l}(z)\right| \leqq \frac{1+\sqrt{\pi}}{\left(z_{0}+{\sqrt{\left.z_{0}^{2}-1\right)^{l+\frac{1}{2}}}}^{2}\right.} \cdot \frac{1}{\sqrt{l+1}} \frac{1}{\left|z^{2}-1\right|^{\frac{1}{4}}} .
$$

We obtain the following representation of $Q_{l}^{\prime}$, for $l$ real and positive, from (A 26) [6]:

$$
Q_{l}^{\prime}(z)=-l(l+1) \int_{0}^{\infty} d u \sinh ^{2} u\left[z+\sqrt{z^{2}-1} \cosh u\right]^{-(l+2)} .
$$

Define

$$
D(y(z))=-\frac{\left(z+\sqrt{z^{2}-1}\right)^{l+2}}{l(l+1)} Q_{l}^{\prime}(z)
$$

With $y$ defined by (A 8). We may write

$$
D(y)=\int_{0}^{\infty} d u \sinh ^{2} u\left\{1+y(\cosh u-1\}^{-(l+2)} .\right.
$$

We again let $x=\cosh u-1$ be the integration variable to obtain

$$
D(y)=\int_{0}^{\infty} d x \sqrt{x(x+2)}\left\{1+x|y| e^{-i \theta}\right\}^{-(l+2)} .
$$


Again we rotate the $x$ integration contour by an angle $\theta$ and divide $D$ into two parts:

$$
\begin{aligned}
D & =\left(D_{1}+D_{2}\right) e^{2 i \theta}, \\
D_{1} & =\int_{0}^{1 /|y|} d t \sqrt{t\left(t+2 e^{-i \theta}\right)}\{1+|y| t\}^{-(l+2)}, \\
D_{2} & =\int_{1 /|y|}^{\infty} d t \sqrt{t\left(t+2 e^{-i \theta}\right)}\{1+|y| t\}^{-(l+2)} .
\end{aligned}
$$

We may change the variable of integration in $D_{1}$ to obtain

$$
D_{1}=\frac{1}{|y|^{2}} \int_{0}^{1} d u \sqrt{u} \sqrt{u+2|y| e^{+i \theta}}\{1+u\}^{-(l+2)} .
$$

We use (A31) and the inequality $\left(u+2|y| e^{i \theta}\right)^{\frac{1}{2}} \leqq \sqrt{3}$ to obtain

$$
D_{1} \leqq \frac{\sqrt{3}}{|y|^{2}} \int_{0}^{1} d u \sqrt{u} e^{-\left(\frac{l+2}{2}\right) u} .
$$

Let us extend the integration in (A41) out to infinity to obtain

$$
\left|D_{1}\right| \leqq \frac{2 \sqrt{6 \pi}}{|y|^{2}} \cdot \frac{1}{(l+2)^{\frac{3}{2}}} .
$$

We may express $D_{2}$ as

$$
D_{2}=\frac{1}{|y|^{2}} \int_{1}^{\infty} d u\left[u\left(u+2|y| e^{-i \theta}\right)\right]^{\frac{1}{2}}\{1+u\}^{-(l+2)} .
$$

We use the inequality $\left|u\left(u+2|y| e^{-i \theta}\right)\right|^{\frac{1}{2}} \leqq 1+u$ to obtain

$$
\begin{aligned}
\left|D_{2}\right| & \leqq \frac{1}{|y|^{2}} \int_{1}^{\infty} d u\{1+u\}^{-(l+1)} \\
& =\frac{T}{l^{2 l}} \cdot \frac{1}{|y|^{2}} \leqq \frac{1}{l|y|^{2}} \cdot \frac{1}{\sqrt{l+1}} .
\end{aligned}
$$

We thus obtain

$$
|D(y)| \leqq \frac{1+2 \sqrt{6 \pi}}{\left.|| y\right|^{2}} \cdot \frac{1}{\sqrt{l+1}} .
$$

Consequently, we have for $l>0$ that

$$
\begin{aligned}
\left|Q_{l}^{\prime}(z)\right| & \leqq \frac{\sqrt{l+1}}{|y|^{2}} \cdot \frac{1+2 \sqrt{6 \pi}}{\left(z_{0}+\sqrt{z_{0}^{2}-1}\right)^{l+2}} \\
& \leqq \frac{\sqrt{l+1}}{\left|z^{2}-1\right|} \frac{10}{\left(z_{0}+\sqrt{\left.z_{0}^{2}-1\right)}\right.} .
\end{aligned}
$$


On the other hand, the relation

$$
Q_{0}^{\prime}(z)=\frac{-1}{z^{2}-1}
$$

allows us to justify the bound (2.36) for $l=0$ as well.

\section{Appendix B}

We are considering a Banach space $\mathscr{B}$ of sequences, $a=\left\{a_{1}, a_{2}, \ldots\right\}$, with the norm

$$
\|a\|=\sup _{l}\left|a_{l}\right| \text {. }
$$

We wish to consider a set $S$ of sequences, such that $S$ is a subset of some finite ball $K_{b}=\{a \mid a \in \mathscr{B},\|a\| \leqq b\}$. We will establish the following compactness criterion:

Lemma. ${ }^{9} S$ is compact in the topology induced by the norm (B 1) if for every number $\varepsilon>0$ there exists an integer $L(\varepsilon)$, such that for every element a of $S$,

$$
\sup _{l>L}\left|a_{l}\right| \leqq \varepsilon
$$

Proof. To show that $S$ is compact if (B2) is met, we construct a finite $\varepsilon$-net for $S$ from elements of $S$. To this end, we first construct a finite $\varepsilon$-net for $S$ from elements of $K_{b}$. For any $\varepsilon>0$, let $N$ be the greatest integer such $\varepsilon(N-1) \leqq 2 b$. Define the $N$ numbers $d_{i}$ by

$$
\left(d_{1}, d_{2}, \ldots, d_{N}\right)=(-b,-b+\varepsilon, \ldots,-b+(N-1) \varepsilon) .
$$

Then for any number $s_{l},-b \leqq s_{l} \leqq g$, there is a $d_{i}$ such that $\left|s_{l}-d_{i}\right| \leqq \varepsilon$. Let $C_{\varepsilon} \subset K_{b}$ be a set of $N^{L}$ sequences, where a sequence in $C_{\varepsilon}$ is denoted by $c\left(n_{1}, n_{2}, \ldots, n_{L}\right)$ and has the form

$$
c\left(n_{1}, n_{2}, \ldots, n_{L}\right)=\left\{d_{n_{1}}, d_{n_{2}}, \ldots, d_{n_{L}}, 0,0, \ldots\right\} .
$$

Here each $n_{i}$ may have any of the values $n_{i}=1,2, \ldots, N$. If $s$ is any element of $S$, there is an element $c$ of $C_{\varepsilon}$ such that $\|S-c\| \leqq \varepsilon$, since with an appropriate choice of the $n_{i}$ we have

$$
\begin{aligned}
\|S-c\| & =\sup \left[\sup _{l \leqq L(\varepsilon)}\left|S_{l}-d_{n_{l}}\right|, \sup _{l>L(\varepsilon)}\left|s_{l}\right|\right] \\
& \leqq \sup [\varepsilon, \varepsilon]=\varepsilon .
\end{aligned}
$$

Consequently, $C_{\varepsilon}$ is a finite $\varepsilon$-net for $S$, composed of elements of $K_{b}$.

9 This lemma is analogous to the more familiar Arzelà-Ascoli equicontinuity criterion for compactness. The latter criterion is appropriate for Banach spaces of functions with a continuous argument. 
We can now find an $\varepsilon$-net $T_{\varepsilon}$ for $S$, composed of elements of $S$. Consider any element $c$ of $C_{\varepsilon / 2}$. If there is at least one element $S$ of $S$ such that $\|c-s\| \leqq \varepsilon / 2$, then put one such element, say $t \in S$, in $T_{\varepsilon}$. Otherwise, go on to another element of $C_{\varepsilon / 2}$. Repeat the procedure for all elements of the finite set $C_{\varepsilon / 2}$. The finite set $T_{\varepsilon}$, consisting of all $t$ selected in this way, is indeed an $\varepsilon$-net for $S$, since for an arbitrary $s \in S$, there is a $c \in C_{\varepsilon / 2}$ such that $\|s-c\| \leqq \varepsilon / 2$, and a corresponding $t \in T_{\varepsilon}$ such that $\|c-t\| \leqq \varepsilon / 2$. Consequently,

$$
\|s-t\| \leqq\|s-c\|+\|c-t\| \leqq \varepsilon .
$$

\section{Appendix C}

Let $k(z)$ be a function analytic in a unifocal ellipse $S\left(z_{0}\right)$ with a continuous derivative on its boundary. Consider its partial wave projection $k_{l}$ for $l=1,2, \ldots$ :

$$
\begin{aligned}
k_{l} & =\frac{1}{2} \int_{-1}^{1} d x P_{l}(x) k(x) \\
& =\frac{1}{2} \int_{-1}^{1} d\left(\int_{-1}^{x} d y P_{l}(y)\right) k(x) .
\end{aligned}
$$

We integrate by parts to obtain

$$
k_{l}=\frac{1}{2}\left|k(1) \int_{-1}^{1} d y P_{l}(y)-\int_{-1}^{1} d x k^{\prime}(x) \int_{-1}^{x} d y P_{l}(y)\right| .
$$

The first term in (C2) vanishes. Furthermore, from the Legendre differential equation we obtain

So

$$
\int_{-1}^{x} d y P_{l}(y)=\frac{\left(x^{2}-1\right)}{l(l+1)} P_{l}^{\prime}(x) .
$$

$$
k_{l}=\frac{-1}{2 l(l+1)} \int_{-1}^{1} d x\left(x^{2}-1\right) P_{l}^{\prime}(x) k^{\prime}(x) \text {. }
$$

We may write $k^{\prime}$ as a Cauchy integral over $\partial S\left(z_{0}\right)$ and use an identity, which is valid for $l>0$ :

$$
\left(1-z^{2}\right) Q_{l}^{\prime}(z)=\frac{1}{2} \int_{-1}^{1} d x \frac{\left(1-x^{2}\right) P_{l}^{\prime}(x)}{z-x} .
$$

This identity is obtained from the Legendre differential equation and (2.14). To verify Eq. (C5), let us differentiate the right side of (C5) with 
respect to $z$, to obtain

$$
-\frac{1}{2} \int_{-1}^{1} d x \frac{d}{d x}\left(\frac{1}{z-x}\right)\left(1-x^{2}\right) P_{l}^{\prime}(x)
$$

We integrate by parts on $x$ to obtain

$$
\begin{aligned}
+\frac{1}{2} \int_{-1}^{1} d x \frac{1}{z-x}\left(\left(1-x^{2}\right) P_{l}^{\prime}(x)\right)^{\prime} & =-\frac{l(l+1)}{2} \int_{-1}^{1} d x \frac{P_{l}(x)}{z-x} \\
& =-l(l+1) Q_{l}(z) .
\end{aligned}
$$

This is the derivative of the left side of (C5), according to Legendre's differential equation. Since for $l>0$ both sides of (C 5) vanish as $|z| \rightarrow \infty$, we have the identity.

It then follows that

$$
k_{l}=\frac{1}{2 \pi i} \frac{1}{l(l+1)} \int_{S\left(z_{0}\right)} d z\left(1-z^{2}\right) Q_{l}^{\prime}(z) k^{\prime}(z) .
$$

One can, consequently, verify (2.27).

\section{References}

1. Atkinson, D., Johnson,P., Warnock, R.: Commun. math. Phys. 28, 133 (1972). References to previous work are given there. See also Ref. 6

2. Atkinson, D., Mahoux, G., Ynduráin, F.: Nucl. Phys. B 54, 263 (1973)

3. Schauder's theorem is discussed in, for example, Kantorowitsch, L.V., Akilow,G.V.: Functional analyse in Normierten Räumen (German translation by Berlin: Akademie Verlag 1964)

4. Bart, G., Johnson, P., Warnock, R.: "Continuum Ambiguity in the Construction of Unitary Analytic Amplitudes from Fixed Energy Scattering Data", IIT preprint, December, 1972

5. Liusternik, L., Sobolev, V.: Elements of Functional Analysis, pp. 138-141. English translation published by New York: F. Ungar, 1961

6. These integral representations are given explicitly in Gradshteyn, I.S., Ryzhik,I.M.: Tables of Integrals, Series and Products, p. 1001. New York: Academic Press, 1965

\section{Atkinson}

Institut voor Theoretische Natuurkunde P. O. Box 800

Groningen, The Netherlands 